\title{
Overexpression of miR-29b Partially Rescued the Osteogenic Differentiation and Osteogenesis Ability of Ovx-BMSCs
}

\author{
Xiaofeng Dai ${ }^{1}$, Gang Deng ${ }^{2}$, Yuanyuan Sun ${ }^{2}$, Bin $\mathrm{Hu}^{3 *}$ and Le Shi ${ }^{1 *}$ \\ ${ }^{1}$ Shanghai Jing-An Dental Clinic, No. 15 Pingxingguan Road, China \\ ${ }^{2}$ Second Dental Clinic, Shanghai Jiao Tong University School of Medicine, China
}

${ }^{3}$ Department of Oral Surgery, Shanghai Jiao Tong University School of Medicine, China.

*Corresponding author: Shanghai Jing-An Dental Clinic, No. 15 Pingxingguan Road, China 2Second Dental Clinic, Shanghai Jiao Tong University School of Medicine, China 3Department of Oral Surgery, Shanghai Jiao Tong University School of Medicine, China.

To Cite This Article: Xiaofeng Dai, Gang Deng, Yuanyuan Sun, Bin Hu and Le Shi, Overexpression of miR-29b Partially Rescued the Osteogenic Differentiation and Osteogenesis Ability of Ovx-BMSCs. Am J Biomed Sci \& Res. 2021 - 12(4). AJBSR.MS.ID.001761.

DOI: 10.34297/AJBSR.2021.12.001761.

Received: 啙 March 22, 2020; Published: 紫 April 08, 2021

\begin{abstract}
Osteoporosis, as a skeletal disorder characterized by gradually reduced bone mineral density, is a severe threat to millions of postmenopausal women. MicroRNAs (miRNAs) are important post-transcriptional factors and play an important role in bone degeneration such as osteoporosis and other bone-related diseases. Here we showed that the expression of miR-29b in bone tissue and bone marrow stromal cells (BMSCs) of Ovx mice is descend and overexpression of miR-29b could partially rescued osteogenic differentiation and bone formation ability of Ovx-BMSCs in vitro and in vivo, respectively. Our studies indicated that miR-29b plays an important role in regulating BMSCs osteogenic differentiation and could contribute to osteoporosis therapy.
\end{abstract}

Keywords: Osteoporosis, miR-29b, BMSCs, Osteogenic differentiation, Osteogenesis

\section{Introduction}

Osteoporosis, the most common bone remodeling disease, is characterized by a systemic impairment of bone mass and microarchitecture and increases the fracture risk of the patient [1]. Moreover, the osteoporotic fracture often causes further severe disability and premature mortality in the aging population due to impaired bone healing abilty $[2,3]$. Worldwide, approximately $40 \%$ of Caucasian postmenopausal women are expected to suffer from osteoporotic fractures [4]. Among the extensive treatment approaches currently used in clinics for treating osteoporosis, most of them are focusing on antiresorptive agents, which exert their therapeutic effect by slowing down the bone resorption rate. However, anti-resorptive strategies mainly focus on preventing on-going damage rather than rebuilding damaged bone tissues
[5]. Bone-anabolic agents including parathyroid hormone (PTH) and and PTH-related peptide (PTHrP) synthetic analogues such as teriparatide and abaloparatide have been used to increased bone formation and reduces bone fracture risk [6-8]. But continuous treatment with PTH may cause bone resorption. Therefore, it is necessary to develop a novel anabolic therapeutic strategy to enhance bone formation.

With the development of regenerative medicine, stem cell-based therapy has attracted much attention for the treatment of bone diseases due to their properties of self-renewal and plasticity [9]. Mesenchymal stem cells (MSCs) such as bone marrow stromal cells (BMSCs) possessing regenerative potential have been considered an ideal cell source for bone formation and repair [10]. However, 
many studies have demonstrated that osteoporosis affecting the osteogenic differentiation of MSCs [11]. Therefore, it is necessary to establish an approach to rescue the osteogenic differentiation and bone formation ability of MSCs under osteoporotic conditions [12].

microRNAs (miRNAs) are a large class of small, non-coding RNAs that function as repressors of gene expression at the level of post-transcriptional regulation [13]. Previous studies have been demonstrated that miRNA network is involved in regulating the process of bone remodeling and also related to osteoporosis phenomenon [14-16]. In this study, a miRNA, namely miR-29b, was identified to be correlated with bone loss in estrogen deficiencyinduced osteoporotic mice. Overexpression of miR-29b could partially rescue the osteogenic differentiation and bone formation ability of osteoporosis-impaired BMSCs. These findings may contribute to stem cell-based bone regeneration under osteoporotic conditions.

\section{Materials and Methods}

\section{Ovariectomy (Ovx)-induced osteoporotic mice model}

All animal experiments were approved by the Animal Care and Use Committee of Anhui Medical University. C57BL/6J mice were used to establish Ovx mice model. At 12 weeks postsurgery, bilateral femurs from Sham-operated or Ovx mice were harvested. Partial of the femurs were analyzed using micro-CT. The osteoporotic Ovx mice model was confirmed by quantification of the bone BV/TV of the distal femurs using micro-CT scan. Partial of the femurs was used to isolate the primary bone marrow stromal cells (BMSCs) for the following studies.

\section{Cell culture}

BMSCs were isolated from the femurs of sham-operated or Ovx mice. The Sham-BMSCs and Ovx-BMSCs were cultured with DMEM medium supplemented with $10 \%$ FBS, $100 \mathrm{U} / \mathrm{ml}$ penicillin, $100 \mathrm{mg} / \mathrm{ml}$ streptomycin, and $2 \mathrm{mM} \mathrm{L}$-glutamine and cultured at $37^{\circ} \mathrm{C}, 5 \% \mathrm{CO} 2$. The culture medium was changed every 2 or 3 days. BMSCs at passage 2 or 3 were used in this study.

\section{MiRNA transfection}

Ovx-BMSCs were transfected with miR-29b, or their respective negative control (NC, Guangdong Ruibo, China) using siPORT NeoFX transfection reagent (Life Technologies, Carlsbad, CA) according to the instruction of the manufacturer. The transfection reagent (total $5 \mu \mathrm{l}$ ) was diluted in $50 \mu \mathrm{l}$ Opti-MEM I (Life Technologies) and mixed with $50 \mathrm{nmol} / \mathrm{L}$ miR-29b, or equal amounts of its respective negative control. The mixture was added into 6-well plates to transfected BMSCs $(1 \times 106$ cells $/ \mathrm{ml})$. After transfection 6 hours, the medium was replaced with fresh culture medium.

\section{Osteogenic differentiation and mineralization assay}

For osteogenic differentiation, cells were cultured with osteogenic differentiation medium containing DMEM, 10\% FBS, $1 \%$ penicillin/streptomycin, $50 \mu \mathrm{g} / \mathrm{mL}$ L-ascorbic acid, $10 \mathrm{mM}$ glycerophosphate and $100 \mathrm{nM}$ dexamethasone. The osteogenic medium was changed every three days. The expression of osteogenesis-related genes including ALP and OCN was determined after 7 days of osteogenic differentiation induction using real-time PCR. Calcium deposition was detected using alizarin red staining (ARS). The cells of each group were fixed with $95 \%$ alcohol for 10 min and then stained with $0.1 \%$ ARS solution (Sigma, St. Louis, MO, USA).

\section{Real-time RT-PCR analysis}

Total mRNA was extracted using TRIzol reagent (Invitrogen). The concentration of total RNA was quantified with a Thermo Scientific NanoDrop ${ }^{\mathrm{TM}} 1000$ ultraviolet-visible spectrophotometer (NanoDrop Technologies, Wilmington, DE). cDNA was synthesized using a Prime-Script TM RT reagent kit (Takara Bio, Shiga, Japan) according to the manufacturer's instructions. The expression of miR-29b and osteogenesis-related marker genes including alkaline phosphatase (ALP) and osteocalcin (OCN) were examined. U6 and Glyceraldehyde-3-phosphate dehydrogenase (GAPDH) were used for normalization of microRNA and mRNA expression levels. All experiments were performed in triplicate. The PCR primer sequences are displayed in (Table 1).

Table 1: Nucleotide sequences for real-time RT-PCR primers.

\begin{tabular}{|c|c|c|c|}
\hline Gene & Primer sequence (5' $\mathbf{-} \mathbf{3}^{\prime}$ ) (forward/reverse) & Product size (bp) & Accession number \\
\hline ALP & AGCTGCCCGCATCCTTAATGTACGTCTTGGAGAGAGCCA & 101 & NM_001287172.1 \\
\hline OCN & GCCCTGACTGCATTCTGCCTCT TCACCACCTTACTGCCCTCCTG & 103 & NM_013414 \\
\hline GAPDH & TGCACCACCAACTGCTTAGGGATGCAGGGATGATGTTC & 177 & NM 001289726.1 \\
\hline
\end{tabular}

\section{In vivo bone formation assay}

Sham-BMSCs, Ovx-BMSCs, Ovx-BMSCs transfected with miR-NC and miR-29b were separately collected and resuspended in FBSfree medium. The collected cells were mixed with hydroxyapatitetricalcium phosphate (HA-TCP) powders (Sigma-Aldrich, St. Louis,
MO, USA), and then the cells/scaffold mixture were subcutaneously transplanted into athymic nude mice. The implants were harvested at 8 weeks post-surgery and decalcified in 10\% Ethylene Diamine Tetraacetic Acid (EDTA) for 2 weeks. The decalcified samples were embedded in paraffin and sectioned at $5 \mathrm{~mm}$. The sections were 
stained with hematoxylin-eosin (HE). Then, the histological images were taken on four consecutive microscopic fields of the section of implants. The new bone formation in transplants was measured as bone tissue area per total area with Image J software.

\section{BMSCs-mediated mouse calvarial bone defect regeneration}

For critical sized calvarial bone defect repair, the animals were anaesthetized by intraperitoneal injection of pentobarbital, and then, a $5 \mathrm{~mm}$ diameter critical-sized defect were created using a trephine bur in osteoporotic mice. Approximately 5.0×106 BMSCs (Ovx+miR-29b or Ovx+miR-NC) were harvested and then dispensed into a $250 \mu \mathrm{l}$ HyStem-HP hydrogel (Glycosan Biosystems, Salt lake City, UT). The gel/cell mixture was transplanted within the defects. To investigate the neo-bone formation, mice were sacrificed at 8 weeks after operation and the calvarial bone samples were harvested and then subjected to micro-CT observations.

\section{Micro-computed tomography (Micro-CT) analysis}

For the calvarial bone analysis, specimens were scanned in scanning mode (voxel size $20 \mu \mathrm{m}, 80 \mathrm{kVp}, 500 \mathrm{~A}$ ) to evaluate the bone volume using a micro-CT system (Siemens AG, Germany). After scanning, three-dimensional images of repaired calvarial bone specimens were reconstructed and the percentage of new bone volume relative to tissue volume was measured using auxiliary software.

\section{Statistics}

The experiment data in this study were presented as the mean \pm standard derivation (SD). Statistical analysis was performed using one-way ANOVA with Tukey's multiple comparison test to assess the difference among multiple experimental groups. Statistical differences between two groups were determined by the Student's t-test. $\mathrm{P}<0.05$ was used considered statistically significant.

\section{Results}

MiR-29 expression pattern in estrogen deficiency-induced osteoporosis mice

A

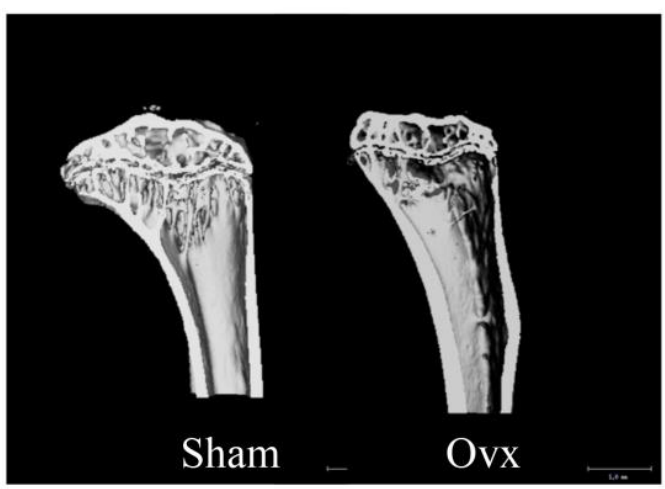

$\mathbf{C}$

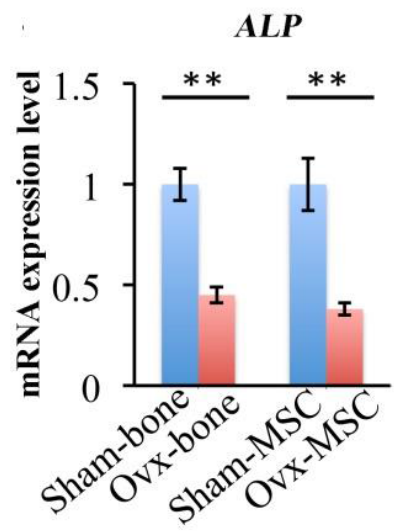

B

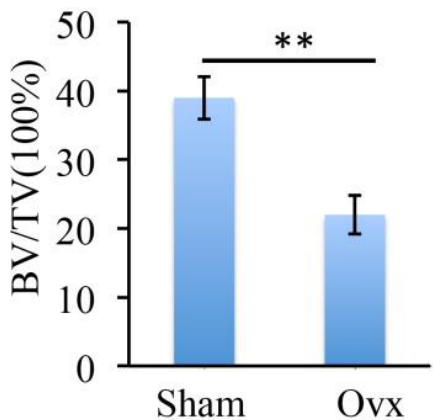

D
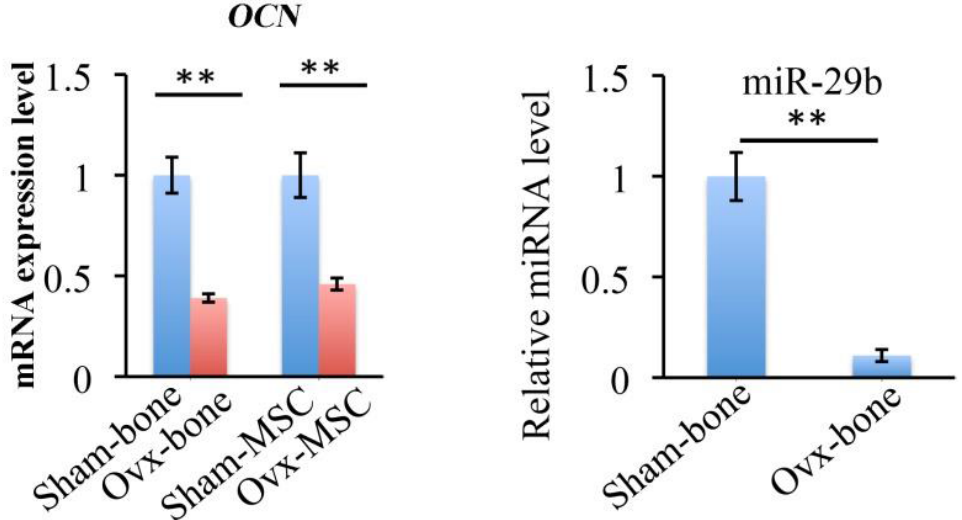

Figure 1(A-D): Characterization of Ovx mice model. (A) Micro-CT images of coronal plane reconstruction of distal femur. (B) Histomorphometric analyze of distal femur cancellous bone by measuring bone volume/total volume (BV/TV). (C) mRNA expression levels of ALP and OCN in bone tissue and BMSCs of Sham and Ovx groups. (D) The expression of miR-29b in bone tissue of Sham and Ovx groups. ${ }^{* *} P<0.01$ 
The osteoporotic mice model was confirmed by quantification of the bone BV/TV of the distal femurs and by analysis of gene expression of osteogenic markers including alkaline phosphatase (ALP) and osteocalcin (OCN) using RT-qPCR. At 8 weeks after postsurgery, micro-CT analysis results indicated that BV/TV of distal femur cancellous bone was significantly reduced in Ovx group than in Sham group (figure 1(A, B)). ALP, as an early differentiation marker, plays an important role in calcium-phosphate mineral formation in bone and OCN is also a marker of mineralization. The expression of ALP and OCN in bone tissue and BMSCs of Ovx mice group was also significantly decreased compared with Sham mice group (figure 1C). miR-29b, had previously been demonstrated that play a role in osteogenic differentiation, exhibited decreased expression in Ovx mice group than in Sham mice group (figure 1D). The role of miR-29b in regulating osteogenic differentiation of OvxBMSCs was analyzed in the following study.

\section{miR-29b overexpression rescued the osteogenic function of Ovx-BMSCs in vitro}

A

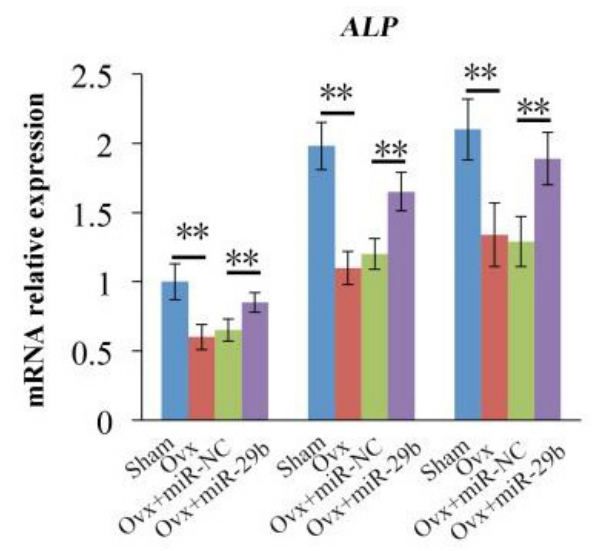

C

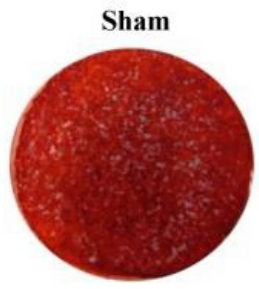

B

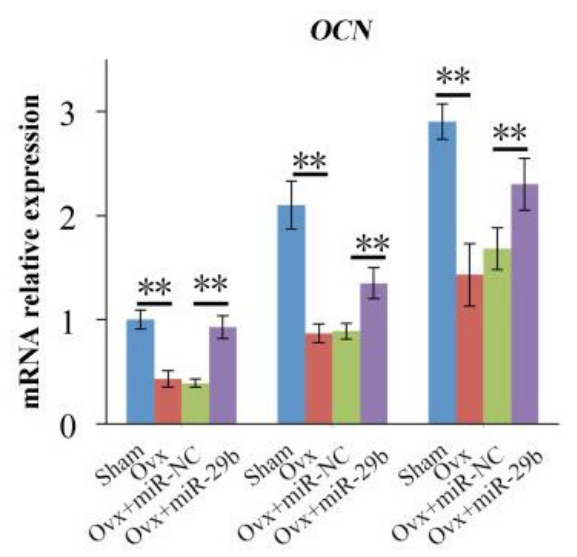

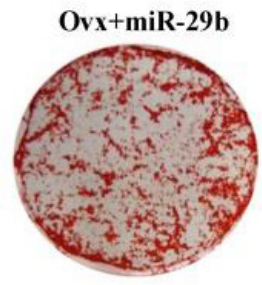

Figure 2(A-C): Overexpression of miR-29b partially rescued osteogenic differentiation of Ovx-BMSCs. (A, B) mRNA expression levels of ALP and $\mathrm{OCN}$ in different groups. (C) Alizarin red S staining of each group. ${ }^{* *} \mathrm{P}<0.01(\mathrm{n}=3)$

To investigate the role of miR-29b in regulating osteogenic differentiation of Ovx-BMSCs, RT-qPCR was performed on day 7 under osteogenic induction. The expression of ALP and OCN were quantified in the groups including Sham-BMSCs, Ovx-BMSCs, and Ovx-BMSCs transfected with miR-NC and miR-29b. The results showed that the expression of ALP and OCN was lower in Ovx-BMSCs group than in Sham-BMSCs group. Overexpression of miR-29b enhanced the expression of ALP and OCN in Ovx-BMSCs compared with Ovx-BMSCs and Ovx-BMSCs transfected with miR-NC. The cells of each group were continuous cultured under osteogenic induction for calcium nodes formation. ARS staining results were consistent with RT-qPCR results. ARS staining was most intense in Sham group compared with all other groups. Although the ARS staining of Ovx+miR-29b was slight weaker than Sham group, the staining revealed a significant increase in the Ovx+miR-29b group compared with Ovx group and Ovx+miR-NC group (figure $2(\mathrm{~A}-\mathrm{C})$ ). These results indicated that overexpression of miR-29b partially rescued the osteogenic function of Ovx-BMSCs in vitro.

\section{miR-29b treatment enhanced ectopic bone formation ability of Ovx-BMSCs in vivo}

To investigate the effects of miR-29b on regulating the BMSCsmediated bone tissue formation in vivo, Sham-BMSCs, Ovx-BMSCs, and Ovx-BMSCs transfected with miR29b or negative control were mixed with HA-TCP scaffolds and subcutaneously transplanted into nude mice. At 8 weeks after operation, the implants were extracted and HE staining was performed for histological analysis. HE staining showed that there was rare hard tissue formation in Ovx-BMSCs group and Ovx-BMSCs transfected with miR-NC group (figure 3A), while new bone tissue formation was increased in miR29b overexpression Ovx-BMSCs group when compared with OvxmiR-NC group and OVX group (figure $3 \mathrm{~A}$ ). The sham group showed the most new bone tissue formation among all the groups (figure $3 \mathrm{~A}$ ). Histomorhpometric analysis of the percentage of new bone area in each group showed the same tendency (figure 3B). 
A

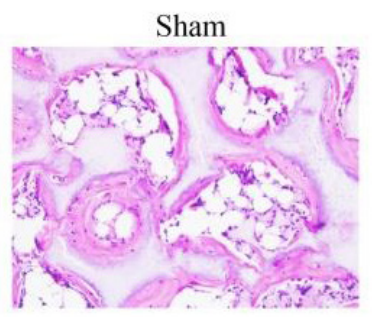

$\mathrm{Ovx}+\mathrm{miR}-\mathrm{NC}$

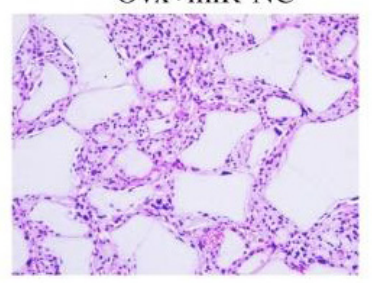

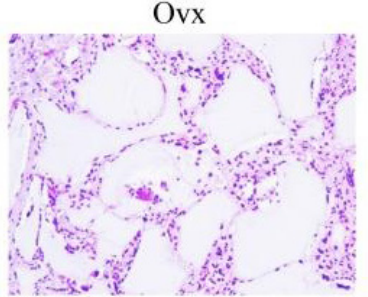

Ovx + miR-29b

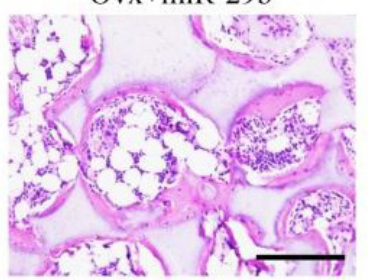

B

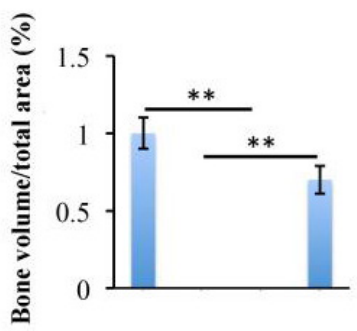

Figure 3(A-B): Overexpression of miR-29b enhanced ectopic bone formation ability of Ovx-BMSCs in vivo. (A) Sham-BMSCs, Ovx-BMSCs and Ovx-BMSCs transfected with miR-NC or miR-29b were mixed with hydroxyapatite-tricalcium phosphate (HA-TCP) and then subcutaneously transplanted into nude mice for 8 weeks. The implants were collected and stained with hematoxylin-eosin (HE). (B) Histomorphometric analysis of the newly formed bone area. Scale bar: $200 \mu \mathrm{m}$.

\section{miR-29b-seeded scaffolds repaired calvarial bone} defects in mice

To further evaluate whether miR-2b can promote bone formation in suit, a 5-mm critical-sized calvarial bone defects was generated in osteoporotic mice. Ovx-miR-NC-BMSCs or Ovx-miR-29bBMSCs were mixed with delivery hydrogel and then transplanted into the defect area. Micro-CT was used to detect the neo-bone formation at two time points day 0 and 12 weeks after operation. The results indicated that the miR-29b transfected group showed more bone tissue regeneration than miR-NC group (figure 4(A-B)). Quantitative study revealed that over $50 \%$ new bone was formed in Ovx+miR-29b group. Meanwhile, only 30\% new bone formation was observed in Ovx+miR-NC group.
A

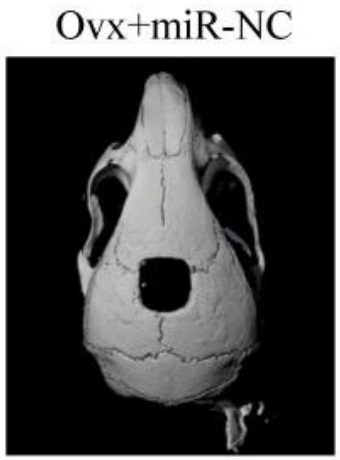

$\mathrm{Ovx}+\operatorname{miR}-29 \mathrm{~b}$

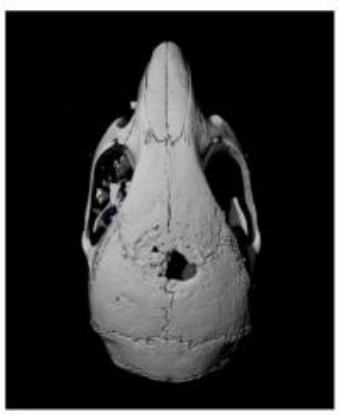

B

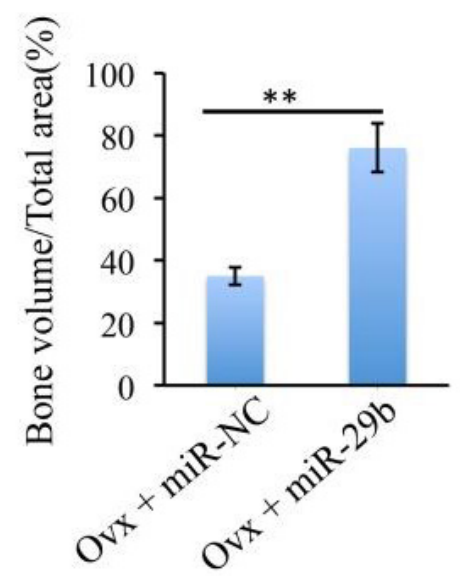

Figure 4: micro-CT analysis. (A) 3D reconstruction images showing the reparative effect of Ovx+miR-NC and Ovx+miR-29b at 12 weeks postsurgery. (B) Bone volume/total volume (BV/TV) analysis in each group.

\section{Discussion}

In this study, we demonstrated that miR-29b is a key regulator of osteoporosis. Descent expression of miR-29b is related to impaired bone formation in osteoporotic mice. Overexpression of miR-29b could partially rescue the osteogenic differentiation and bone formation ability of osteoporosis-impaired BMSCs. MicroRNAs are a large class of small non-coding RNAs that are also closely related to bone development. Plenty of miRNAs such as miR-133 are demonstrated to be involved in osteogenesis related signaling pathways [17]. miRNA mimic or miRNA inhibitors has been 
considered as candidates to be used as therapies for disease as consequence of osteoblasts and osteoclasts malfunction [18]. The miR-29 family contains three mature miRNAs including miR-29a, miR-29b and miR-29c [19]. Previous study has been demonstrated that miR-29b is a positive regulator of osteogenic differentiation by directly down-regulating osteogenic differentiation inhibitors [20]. Thus, we investigated the potential role of miRNA in regulating bone disease osteoporosis.

In this study, we first demonstrated that the expression of miR$29 \mathrm{~b}$ both in bone tissue and bone marrow derived BMSCs was downregulated in Ovx-induced osteoporotic mice when compared with Sham-mice. The expression of osteogenic related genes including ALP and OCN showed the same tendency in Ovx-mice and Shammice. Then, we investigated the potential of miR-29b to restore the osteogenic capacity of osteoporosis-impaired BMSCs. Our results showed that overexpression of miR-29b could enhance the expression of osteogenic differentiation related markers including ALP and OCN in Ovx-BMSCs. The calcium nodes deposition also increased in Ovx+miR29-b group. Furthermore, overexpression of miR-29b also enhanced ectopic bone formation of Ovx-BMSCs in vivo.

Osteoporosis, especially postmenopausal osteoporosis, has a severe influence in aging population [21]. In current, several drugs such as bisphosphonates, calcitonin and so on have been used to slow the process of bone loss [22-23]. Moreover, administration of stem cells could prevent or reverse the osteoporosis and reduce the susceptibility of bone fractures. In this study, we also investigated the in suit bone formation ability of Ovx-BMSC transfeted with miR29b using a mouse critical-sized calvarial defect model. Micro-CT analysis showed that miR-29b significantly enhanced the bone regeneration ability of Ovx-BMSCs. Over $50 \%$ bone defect area was repaired by the newly formed bone tissue.

\section{Conclusion}

This study indicated that overexpression of miR-29b could partially rescued the osteogenic differentiation ability and osteogenesis ability of Ovx-BMSCs both in vitro and in vivo. These findings suggested that miR-29b may be a promising therapeutic target for potent osteoporosis and osteoporotic fracture treatments.

\section{Conflicts of Interest}

The authors indicated no potential conflicts of interest.

\section{References}

1. Curtis EM, Moon RJ, Harvey NC, Cooper C (2017) The impact of fragility fracture and approaches to osteoporosis risk assessment worldwide. Bone 104: 29-38

2. Bottani M, Banfi G, Lombardi G (2019) Perspectives on mirnas as epigenetic markers in osteoporosis and bone fracture risk: A step forward in personalized diagnosis. Frontiers in genetics 10: 1044
3. Chandran S, John A (2019) Osseointegration of osteoporotic bone implants: Role of stem cells, silica and strontium - a concise review. Journal of clinical orthopaedics and trauma 10(Suppl 1): S32-s36.

4. Jackson RD, Mysiw WJ (2014) Insights into the epidemiology of postmenopausal osteoporosis: The women's health initiative. Seminars in reproductive medicine 32(6): 454-462.

5. Shibahara T (2019) Antiresorptive agent-related osteonecrosis of the jaw (aronj): A twist of fate in the bone. The Tohoku journal of experimental medicine 247(2): 75-86.

6. Bhattacharyya S, Pal S, Chattopadhyay N (2018) Targeted inhibition of sclerostin for post-menopausal osteoporosis therapy: A critical assessment of the mechanism of action. European journal of pharmacology 826: 39-47.

7. Esbrit P, Alcaraz MJ (2013) Current perspectives on parathyroid hormone (pth) and pth-related protein (pthrp) as bone anabolic therapies. Biochemical pharmacology 85(10): 1417-1423.

8. Tella SH, Kommalapati A, Correa R (2017) Profile of abaloparatide and its potential in the treatment of postmenopausal osteoporosis. Cureus 9(5): e1300

9. Ghasroldasht MM, Irfan-Maqsood M, Matin MM, Bidkhori HR, NaderiMeshkin H, et al. (2014) Mesenchymal stem cell based therapy for osteodiseases. Cell biology international 38(10): 1081-1085.

10. Knight MN, Hankenson KD (2013) Mesenchymal stem cells in bone regeneration. Advances in wound care 2(6): 306-316.

11. Tan J, Xu X, Tong Z, Lin J, Yu Q et al. (2015) Decreased osteogenesis of adult mesenchymal stem cells by reactive oxygen species under cyclic stretch: A possible mechanism of age-related osteoporosis. Bone research 3: 15003.

12. Kiernan J, Davies JE, Stanford WL (2017) Concise review: Musculoskeletal stem cells to treat age-related osteoporosis. Stem cells translational medicine 6(10): 1930-1939.

13. Dernowsek JA, Pereira MC, Fornari TA, Macedo C, Assis AF, et al. (2017) Posttranscriptional interaction between mir-450a-5p and mir-28-5p and stat1 mrna triggers osteoblastic differentiation of human mesenchymal stem cells. Journal of cellular biochemistry 118(11): 4045-4062.

14. Hesse E, Taipaleenmaki H (2019) Micrornas in bone metastasis. Current osteoporosis reports 17(3): 122-128.

15. Xie Y, Chen Y, Zhang L, Ge W, Tang P (2017) The roles of bone-derived exosomes and exosomal micrornas in regulating bone remodelling. Journal of cellular and molecular medicine 21(5): 1033-1041.

16. Zeng Q, Wang Y, Gao J, Yan Z, Li Z, et al. (2019) Mir-29b-3p regulated osteoblast differentiation via regulating igf- 1 secretion of mechanically stimulated osteocytes. Cellular \& molecular biology letters 24(11).

17. Lv H, Sun Y, Zhang Y (2015) Mir-133 is involved in estrogen deficiencyinduced osteoporosis through modulating osteogenic differentiation of mesenchymal stem cells. Medical science monitor: international medical journal of experimental and clinical research 21: 1527-1534.

18. Frohlich LF (2019) Micrornas at the interface between osteogenesis and angiogenesis as targets for bone regeneration. Cells 8(2): 121.

19. Schmitt MJ, Margue C, Behrmann I, Kreis S (2013) Mirna-29: A microrna family with tumor-suppressing and immune-modulating properties. Current molecular medicine 13(4): 572-585.

20. Li Z, Hassan MQ Jafferji M, Aqeilan RI, Garzon R, et al. (2009) Biological functions of mir-29b contribute to positive regulation of osteoblast differentiation. The Journal of biological chemistry 284(23): 1567615684. 
21. Xie Y, Zhang L, Xiong Q Gao Y, Ge W, Tang P (2019) Bench-to-bedside strategies for osteoporotic fracture: From osteoimmunology to mechanosensation. Bone research 7:25.

22. Compston J (2020) Practical guidance for the use of bisphosphonates in osteoporosis. Bone: 115330.
23. Kotak DJ, Devarajan PV (2020) Bone targeted delivery of salmon calcitonin hydroxyapatite nanoparticles for sublingual osteoporosis therapy (slot). Nanomedicine: nanotechnology, biology, and medicine 24: 102153. 